\title{
Frecuencia de casos de úlceras de presión y factores asociados a su desarrollo en adultos mayores hospitalizados en servicios de Medicina de un hospital general.
}

Frequency of pressure ulcers and its related factors in elderly patients admitted to the Medicine wards at a general hospital.

Peralta Vargas Carmen Eliana ${ }^{1}$, Varela Pinedo Luis Fernando ${ }^{2}$, Gálvez Cano Miguel ${ }^{3}$.

\section{RESUMEN}

Objetivo: Determinar la frecuencia de casos de úlcera de presión y los factores asociados más importantes para su aparición en pacientes adultos mayores hospitalizados en el servicio de medicina de un hospital general. Material $\boldsymbol{y}$ métodos: Estudio prospectivo, observacional. Se estudiaron a todos los pacientes mayores de 60 años, hospitalizados en el servicio de medicina del Hospital Nacional Cayetano Heredia, entre diciembre de 2006 y abril de 2007. Se evaluaron diferentes variables demográficas, clínicas, así como diversos síndromes geriátricos; en dos grupos de pacientes, quienes desarrollaron úlcera de presión y los que no desarrollaron úlcera de presión. Se utilizó chi cuadrado y el análisis multivariado para determinar asociación estadística. Resultados: Se incluyeron 463 adultos mayores, la frecuencia de casos de úlcera de presión fue 11,4\%. Las variables que estuvieron asociadas con el desarrollo de úlcera de presión, con una significancia estadística en el análisis univariado fueron: edad mayor de 70 años, dependencia parcial o total, deterioro cognitivo moderado o severo, incontinencia urinaria, malnutrición, riesgo moderado y alto para desarrollar úlcera de presión según escala de Norton, neumonía y accidente cerebrovascular como diagnósticos de ingreso, uso de catéter vesical, sujeción mecánica, dispositivos externos locales en zonas de prominencias óseas, la presencia de cuidador y el desarrollo de infección pulmonar intrahospitalaria. Luego del análisis multivariado solo: malnutrición (OR =6,87; IC 95\%, 1,38-34,06 p=0,01) y sujeción mecánica (OR=8,09; IC 95\%, 2,47-26,41 p=0,001) fueron los factores de riesgo. Conclusiones: La úlcera de presión es una condición frecuente en el adulto mayor hospitalizado. Los factores asociados a su aparición fueron malnutrición y sujeción mecánica.(Rev Med Hered 2009;20:16-21).

PALABRAS CLAVE: Ulcera de presión, factores asociados, adultos mayores.

1 Médico Residente de Medicina Interna del Hospital Nacional Cayetano Heredia. Facultad de Medicina Alberto Hurtado, Universidad Peruana Cayetano Heredia. Lima, Perú.

2 Director del Instituto de Gerontología de la Universidad Peruana Cayetano Heredia. Lima, Perú.

3 Médico Asistente del Servicio de Geriatria, Departamento de Medicina. Hospital Nacional Cayetano Heredia. Lima, Perú. 


\section{SUMMARY}

Objective: To assess the frequency of pressure ulcers and its related factors in elderly patients admitted to the Medicine wards at a national hospital. Material and methods: Prospective and observational study. We evaluated patients older than 60 years of age who were admitted to Medical wards of the Hospital Nacional Cayetano Heredia between December 2006 and April 2007. We assess clinical and demographic variables and geriatrics syndromes in two groups of elderly patients according with the presence of pressure ulcers or not. We performed chi-square and multivariate analyses for detecting statistic association. Results: We studied 463 elderly patients, and the frequency of pressure ulcers was $11.4 \%$. The following variables were associated with pressure ulcers: patients older than 70 years old, partial or total dependency, moderate and severe cognitive dysfunction, urine incontinence, malnutrition, moderate risk and higher risk according Norton scale, pneumonia and stroke as entry diagnosis, use of bladder catheter, physical restraints, use of pressure-reducing cushions and heel protectors, caregiver support and the presence of in-hospital pneumonia. In the multivariable analyses, only malnutrition (OR=6.87; IC 95\%, 1.38-34.06, $\mathrm{p}=0.01)$ and physical restraints $(\mathrm{OR}=8.09$; IC 95\%, 2.47-26.41, $\mathrm{p}=0.001)$ were significant associated factors.Conclusions: Pressure ulcers are frequent in hospitalized elderly patients. The associated factors for pressure ulcers were malnutrition and physical restraints.(Rev Med Hered 2009;20:16-21).

KEY WORDS: Pressure ulcers, associated factors, elderly.

\section{INTRODUCCIÓN}

La úlcera de presión (UP) es una condición frecuente en el adulto mayor y es producida por la prolongada presión ejercida por un objeto externo sobre prominencias óseas, provocando ulceración y necrosis del tejido involucrado. La OMS en el 2004 reconoció que sigue siendo alta la incidencia de este problema de salud: $55 \%$ (1). A nivel de hospicios y de residencias geriátricas norteamericanas, existe una incidencia al año de $13 \%$. Su reconocimiento es importante, puesto que se han demostrado repercusiones en diferentes ámbitos: en la salud y la calidad de vida de quienes la padecen, en sus cuidadores, en el consumo de recursos para el sistema de salud, pudiendo incluso alcanzar responsabilidad legal al ser considerado en muchas situaciones como un problema evitable (2).

El impacto de la úlcera de presión en el adulto mayor hospitalizado estriba en una estancia hospitalaria cinco veces mayor que en pacientes sin esta condición, un incremento en la mortalidad, un aumento significativo de complicaciones infecciosas y comorbilidad, y un incremento en los costos de manejo, estimándose en 489 dólares por úlcera, con un tiempo medio de tratamiento de 116 días $(3,4)$.

El hecho de que aparezca una UP depende de una compleja interacción entre factores relacionados con el paciente, condiciones de comorbilidad y factores tisulares (5); así como circunstancias relacionadas a la hospitalización y administración de medicamentos (1).
En teoría, las personas con riesgo elevado para el desarrollo de UP pueden ser identificadas mediante las escalas de Norton o Braden, sin embargo a pesar de ellas, su incidencia sigue en aumento pudiendo ser explicado por la inefectividad en la elaboración y ejecución de medidas terapéuticas y preventivas (6).

Actualmente, en el Perú, los adultos mayores representan el $8,79 \%$ de la población y se estima que para el 2025 será de 12,44\% (1,7); por lo tanto es de esperar que cada día el médico deba afrontar más casos de UP en su práctica profesional. La poca información que se tiene sobre esta condición en el ámbito nacional, nos motivaron a realizar un estudio para determinar la frecuencia de casos de UP en los pacientes adultos mayores hospitalizados en los servicios de medicina y describir los principales factores asociados a su aparición.

\section{MATERIAL Y MÉTODOS}

Estudio prospectivo, longitudinal, observacional. Se incluyeron en el estudio todos los pacientes adultos mayores hospitalizados en los Servicios de Medicina del Hospital Nacional Cayetano Heredia (HNCH), desde diciembre de 2006 hasta abril de 2007.

Los criterios de exclusión fueron: pacientes adultos mayores que se hospitalizaban en UCI a su ingreso, pacientes o familiares que se negaron a firmar el consentimiento informado para participar en el estudio, pacientes que presentaron UP antes del ingreso y 
pacientes que usaron colchón de aire en su hospitalización. Luego se establecieron dos grupos a comparar: uno de adultos mayores con UP y otro sin esta condición.

El estudio fue aprobado por los Comités de Ética y de Investigación de la Universidad Peruana Cayetano Heredia y del HNCH.

Se registraron las siguientes variables: edad, sexo, estado cognitivo (8), nivel funcional (9), estado nutricional (10), incontinencia urinaria, estancia hospitalaria, el diagnóstico que motivó la hospitalización, número de enfermedades previas, dispositivos externos utilizados en el paciente para disminuir la presión ejercida en la piel sobre prominencias óseas (rodetes y guantes estériles inflados con agua), UP previa, y presencia de vía periférica endovenosa, cateter vesical, sujeción mecánica, presencia de cuidadores y complicación intrahospitalaria.

Para diagnosticar UP, definida por la presencia de enrojecimiento, erosión epidérmica del TCSC, músculo, tendón o hueso en un área anatómica específica, se realizó una evaluación inicial dentro de las primeras 24 horas de su hospitalización, y luego cada 7 días con el fin de documentar la presencia de úlcera de presión. El seguimiento culminaba al encontrar un caso de UP o al alta, sin realizarse intervenciones adicionales a las tomadas por los servicios. Previo al estudio se realizó un estudio piloto, cuyos resultados sirvieron para hacer ajustes en los procedimientos.

\section{Análisis estadístico}

Los datos fueron procesados y analizados mediante el programa SPSS 12,0 para Windows. Se hallaron frecuencia y porcentajes para las variables discretas y medias y desviación standard (SD) para las edades. Para el análisis de los datos se utilizó la prueba t de Student de dos colas para la comparación de las variables continuas, la prueba de Chi-Cuadrado o la prueba exacta de Fisher para la comparación de las variables discretas y el test de Mantel-Haenszel para el análisis de tendencias lineares en proporciones en el caso de variables multicategóricas, considerándose una diferencia estadísticamente significativa un $\mathrm{p}<0,05$. Para medir la relación factor/enfermedad se determinó la razón de riesgo (OR) y para controlar el posible efecto de variables de confusión, se realizó un análisis multivariado de regresión logística, considerándose sólo las variables que en el modelo univariado alcanzaron un valor de $\mathrm{p}<0,05$.

\section{RESULTADOS}

Durante el período de estudio fueron hospitalizados 463 pacientes mayores de 60 años, cuyo promedio de edad fue de 75,08 \pm 9 ,29 años (rango de 60-98 años), $51,6 \%$ (239 pacientes) era de sexo masculino. El 30,7\% (142 pacientes) se hospitalizó en el servicio de medicina B, 27,0\% (125 pacientes) en el servicio de medicina A y 42,3\% (196 pacientes) en el servicio de medicina emergencia. Los datos de los pacientes estudiados se muestran en la tabla $\mathrm{N}^{\circ} 1$.

La frecuencia de casos de UP en los adultos mayores hospitalizados fue de 11,4\% (53 pacientes).

La presencia de complicaciones intrahospitalarias fue más frecuente en el grupo de pacientes con UP respecto al grupo control (49,1\% vs $21,0 \%$; OR=3,62; IC 95\%, 2,01-6,53, p=0,0001). Se desarrolló neumonía intrahospitalaria en $20,8 \%$ de los pacientes con UP y en $4,4 \%$ de los pacientes sin ella (OR=5,70; IC 2,52 $12,88, \mathrm{p}=0,0001)$. No se encontró diferencia en la mortalidad en ambos grupos (18,9\% en pacientes con UP vs 12,4\%; OR=1,63; IC 95\%, 0,77-3,45 p=0,19).

Las variables asociadas al desarrollo de UP en el análisis univariado fueron: la edad, nivel funcional de dependencia parcial o total previo a la hospitalización, deterioro cognitivo moderado y severo, presencia de incontinencia urinaria, riesgo de malnutrición y malnutrición, riesgo moderado o alto según la escala de Norton, diagnóstico de neumonía y de accidente cerebrovascular al ingreso, uso de cateter vesical, presencia de cuidador, sujeción mecánica y el uso de dispositivos externos $(\mathrm{p}<0,05)\left(\right.$ Tabla $\left.\mathrm{N}^{\circ} 2\right)$.

Tabla $N^{\circ} 1$. Características iniciales de los pacientes $(n=463)$.

n

$\%$

Edad años 75,08 \pm 9,29 años(rango 60-98)

$\begin{array}{lrr}\text { Sexo masculino } & 239 & (51,60 \%) \\ \text { Funcionalmente independiente } & 306 & (66,09 \%) \\ \text { Sin deterioro cognitivo } & 231 & (49,89 \%) \\ \text { Sin incontinencia urinaria } & 221 & (47,73 \%) \\ \text { Sin diagnósticos previos } & 83 & (17,92 \%) \\ \text { Sin riesgo de malnutrición } & 146 & (31,53 \%) \\ \text { Con bajo riesgo para desarrollar úlcera } & & \\ \text { de presión, según escala Norton } & 343 & (74,08 \%)\end{array}$


En el análisis multivariado de regresión logística solo malnutrición (OR =6,87; IC 95\%, 1,38-34,06, $\mathrm{p}=0,01)$ y sujeción mecánica ( $\mathrm{OR}=8,09$; IC 95\%, 2,4726,41, $\mathrm{p}=0,001$ ) se asociaron con la aparición de UP en el adulto mayor hospitalizado.

\section{DISCUSIÓN}

A pesar de las medidas preventivas la evidencia foránea refiere que la incidencia de UP ha incrementado en 55\% desde $1983(3,4,5)$, pudiendo explicarse por: el incremento de la población adulta mayor y por la complejidad de su patología.

En nuestro trabajo, la frecuencia de casos de UP en pacientes adultos mayores durante su hospitalización fue $11,4 \%$, mayor al encontrado (entre $3 \%$ y $11 \%$ ) en la revisión sistemática de Reddy y col (5), sin embargo al comparar con estudios nacionales, fue menor al 57\% encontrado por Pinedo (11) y, al 16\% encontrado por Izquierdo (12).

La edad avanzada ha sido descrita como un factor de riesgo para la aparición de UP $(3,5)$. Pergener $(13)$ encontró que una mayor edad constituye uno de los factores principales de riesgo de UP. Chigne (4), encontró una relación directa y significativa entre edad y riesgo alto de UP. Nosotros encontramos que una edad mayor o igual a 70 años estuvo asociada con mayor riesgo de UP en el análisis univariado, esto podría ser explicado por los cambios tróficos en la piel y estructurales en la masa muscular.

La primera causa de hospitalización en adultos mayores está representada por las infecciones, sobretodo neumonía, infección del tracto urinario y

Tabla ${ }^{\circ} 2$. Factores de riesgo para el desarrollo de úlceras de presión (Análisis univariado).

\begin{tabular}{|c|c|c|c|c|}
\hline Variable & $\begin{array}{c}\text { Ulcera de } \\
\text { presión (\%) } \\
n=53\end{array}$ & $\begin{array}{c}\text { No úlcera de } \\
\text { presión (\%) } \\
n=410\end{array}$ & OR (IC al 95\% & $p$ \\
\hline Sexo masculino & 39,6 & 53,2 & $0.5(0.3-1.1)$ & 0,066 \\
\hline \multicolumn{5}{|l|}{ Grupo de edad } \\
\hline $60-69$ a & 11,3 & 35,4 & 1,0 & \\
\hline $70-79$ a & 43,4 & 34,4 & $3,9(1,5-9,9)$ & 0,004 \\
\hline 80-89 а & 22,6 & 23,7 & $2,9(1,1-8,2)$ & 0,034 \\
\hline 90-99 а & 22,6 & 6,6 & \multicolumn{2}{|c|}{$10,7(3,7-31,1) 0,0001$} \\
\hline \multicolumn{5}{|l|}{ Katz } \\
\hline Independiente & 43,4 & 68,8 & 1,0 & \\
\hline Dependiente parcial & 35,8 & 23,4 & $2,4(1,2-4,6)$ & 0,008 \\
\hline Dependiente total & 20,8 & 7,3 & $4,5(2,0-10,1)$ & 0,0001 \\
\hline \multicolumn{5}{|l|}{ Pfeiffer } \\
\hline Normal & 40,7 & 67,7 & 1,0 & \\
\hline Leve & 14,8 & 13,8 & $1,7(0,5-5,8)$ & 0,343 \\
\hline Moderado & 25,9 & 12,6 & $3,4(1,2-9,3)$ & 0,017 \\
\hline Severo & 18,5 & 5,8 & $5,2(1,6-16,7)$ & 0,005 \\
\hline Incontinencia urinaria & 67,9 & 50,2 & $2,1(1,1-3,8)$ & 0,017 \\
\hline \multicolumn{5}{|l|}{ Diagnósticos previos } \\
\hline Ninguno & 24,5 & 17,1 & 1,0 & \\
\hline Un diagnóstico & 37,7 & 34,9 & $0,7(0,3-1,6)$ & 0,46 \\
\hline Más de uno & 37,7 & 48,0 & $0,5(0,2-1,1)$ & 0,11 \\
\hline \multicolumn{5}{|l|}{ Estado nutricional } \\
\hline No riesgo de malnutrición & 5,7 & 34,9 & 1,0 & \\
\hline Riesgo de malnutrición & 26,4 & 38,3 & $4,2(1,2-15,1)$ & 0.025 \\
\hline Malnutrición & 67.9 & 26,8 & $15,6(4,6-51,9)$ & 0,0001 \\
\hline \multicolumn{5}{|l|}{ Escala de Norton } \\
\hline Bajo riesgo & 37,7 & 78,8 & 1,0 & \\
\hline Riesgo moderado & 26,4 & 8,0 & $6,8(3,1-14,8)$ & 0,0001 \\
\hline Riesgo alto & 35,8 & 13,2 & $5,6(2,8-11,3)$ & 0,0001 \\
\hline \multicolumn{5}{|l|}{ Diagnóstico de ingreso } \\
\hline Neumonía & 35,8 & 19,0 & $2,3(1,2-4,3)$ & 0,006 \\
\hline Enfermedad cerebrovascular & 18,9 & 8,0 & $2,6(1,2-5,7)$ & 0,013 \\
\hline Vía periférica & 98,1 & 92,0 & $4,5(0,6-33,9)$ & 0,06 \\
\hline Sonda Vesical & 50,9 & 35,6 & $1,8(1,1-3,3)$ & 0,03 \\
\hline Presencia de cuidador & 34,0 & 19,8 & $2,1(1,1-3,8)$ & 0,02 \\
\hline Sujeción mecánica & 62,3 & 21,9 & $5,8(3,2-10,7)$ & 0,0001 \\
\hline Dispositivos externos & 45,3 & 11,0 & $6,7(3,5-12,5)$ & 0,0001 \\
\hline
\end{tabular}


sepsis $(14,15)$. En nuestro estudio los diagnósticos más frecuentes de ingreso y que se asociaron al desarrollo de UP fueron neumonía $(\mathrm{p}=0,006)$ y accidente cerebrovascular $(p=0,01)$. Este hecho, podría ser explicado por el impacto que tienen estas dos enfermedades en el estado general y en la movilidad de este grupo etáreo.

Un resultado que llamó la atención en el análisis univariado, fue que la presencia de cuidadores y el uso de dispositivos externos, estuvieron asociados con el desarrollo de UP. Estas eran consideradas como medidas de protección y cuidado para prevención de UP en otras realidades. Este hecho ha generado nuevas expectativas de prevención y planes de capacitación a los cuidadores, con el fin de disminuir los factores de riesgo de UP.

Otras variables asociadas al desarrollo de UP en el adulto mayor hospitalizado encontradas en nuestro estudio, fueron: deterioro cognitivo moderado y severo, un nivel funcional de dependencia parcial o total, riesgo moderado y alto para úlcera de presión según la escala de Norton, neumonía y accidente cerebrovascular como diagnósticos de ingreso, ser portador de sonda vesical, la presencia de incontinencia urinaria; tal y como se describe en otros estudios (11); además de desarrollo de neumonía como complicación intrahospitalaria (16). Esto se explicaría por el impacto de estos factores en la movilidad y en el estado general del adulto mayor (17).

El factor nutricional es un importante factor de riesgo de UP. Se han descrito anomalías del peso corporal, valores anormales del pliegue tricipital y del IMC (4) e hipoalbuminemia. En un estudio realizado en Cuba, la malnutrición estuvo presente entre 41 y $61 \%$ de los adultos mayores hospitalizados (18). En nuestro estudio encontramos que $67,9 \%$ de adultos mayores con UP tuvieron malnutrición y estuvo asociado a mayor riesgo de aparición de UP en el análisis univariado y multivariado; similar a lo que reportaron Lipshitz y Casimiro $(17,19)$.

Lo que más nos llamó la atención fue que la sujeción mecánica, una acción que se hace de manera rutinaria en el servicio de medicina y que no es usualmente una indicación médica, tenga un impacto importante en la aparición de UP al realizarse el análisis multivariado (OR= 8,09 IC: 2,47-26,41; $\mathrm{p}=0,001$ ); por ello es importante desarrollar un plan de atención preventivo y reemplazar esta acción por otras medidas (camas con baranda).
El ser un estudio prospectivo, longitudinal y observacional fue una de las fortalezas del trabajo, así como el hecho de tener un seguimiento cercano y un número adecuado de pacientes. Una debilidad del estudio realizado fue que algunas de las variables significativas en el análisis univariado, no lo fueron en el análisis multivariado probablemente por el número pequeño de pacientes con úlcera de presión, que impidió demostrar que esas variables tuvieran significancia estadística.

En conclusión, la frecuencia de casos de UP en los adultos mayores hospitalizados en el servicio de medicina del Hospital Nacional Cayetano Heredia durante el período de estudio fue $11,4 \%$. Las variables asociadas con un mayor riesgo de aparición de UP fueron: malnutrición y sujeción mecánica.

\section{Correspondencia:}

\section{Carmen Eliana Peralta Vargas}

Instituto de Gerontología

Universidad Peruana Cayetano Heredia.

Av. Honorio Delgado N 430. San Martín de Porres. Lima Perú.

Correo electrónico: cepv41236@yahoo.es

\section{REFERENCIAS BIBLIOGRÁFICAS}

1. Varela L, Chávez H, Herrera A, Ortiz P, Chigne O. Valoración geriátrica integral en adultos mayores hospitalizados a nivel nacional. Diagnóstico 2003; 42: 73-80.

2. Maklebust J, Sieggreen M, Sidor D, et al. Pressure ulcers: the great insult. Nurs Clin North Am 2005; 40:365-89.

3. Instituto Nacional de Estadística e Informática (INEI). Perfil sociodemogràfico de la tercera edad. Perú 2005. URL disponible en: http:// www.inei.gob.pe/biblioineipub/bancopub.htm. (Fecha de acceso: 8 de agosto de 2006)

4. Chigne O, Varela L, Chávez H. Aplicación de la escala de Norton para evaluar riesgo de úlceras por presión en pacientes adultos mayores hospitalizados. Bol Soc Peru Med Interna 2000; 13: 78-84.

5. Reddy M, Sudeep S, Rochon P. Preventing pressure ulcers: a systematic review. JAMA. 2006; 296:974-84.

6. Blümel J, Tirado K, Schile C, Schönffeldt G, Sarrá S. Validez de la escala de Braden para predecir úlceras por presión en población femenina. Rev Med Chile 2004; 132: 595-600.

7. Varela L, Chávez H, Herrera A, Méndez F, Gálvez M. Perfil del adulto mayor Perú -INTRA II 2004. 
Desarrollando Respuestas Integradas de Sistemas de Cuidados de Salud para una población en rápido envejecimiento. URL disponible en: http:// www.upch.edu.pe/vrinve/gerontología/pdfs/ prueba-intrall.pdf (Fecha de acceso: 10 de agosto de 2006)

8. Pffeifer E. A short portable mental status questionnaire for the assessment of organic brain deficit in elderly. J Am Geriatr Soc 1975; 23: 433-41.

9. Katz F, Dube D, Calkins E. Use of a structures functional assessment format in a geriatric consultive service. J Am Geriatr Soc 1985; 33: 681-6.

10. Gómez M, Gonzáles F, Sánchez C. Estudio del estado nutricional en la población anciana hospitalizada. Nutr Hosp 2005; 20: 286-92.

11. Pinedo J. Aplicación de la escala de valoración de Braden y presencia de úlceras por presión en pacientes de alto y moderado riesgo en el Servicio de Medicina del Hospital Nacional Cayetano Heredia. Tesis para obtener el grado de Licenciada en Enfermería. Lima, Perú. Universidad Peruana Cayetano Heredia, 1999. 27 pp.

12. Izquierdo F. Relación entre riesgo e incidencia de úlcera por presión en el adulto mayor hospitalizado en el servicio de medicina del Hospital Nacional Cayetano Heredia. Tesis para obtener el titulo de Especialista en Enfermería Intensivista. Lima, Perú.Universidad Nacional Mayor de San Marcos, 2005.73 pp.

13. Perneger T, Rae A, Gaspoz J, et al. Screening for pressure ulcer risk in an acute care hospital: development of a brief scale. J Clin Epidemiol 2002;
55: 498-504.

14. Varela L, Sillicani A, Chávez H, Chigne O. Valoración geriátrica integral: propuesta de addendum a la historia clínica. Diagnóstico 2000; 39: $135-47$.

15. Ortiz P, Chávez J, Varela L. Delirio en el paciente adulto mayor hospitalizado: presentación clínica y morbimortalidad. Rev Soc Peru Med Interna 2001; 14: $184-90$

16. Allman R, Goode P, Burst N, Bartolucci A, Thomas D. Pressure ulcers, hospital complications, and disease severity: impact on hospital cost and length of stay. Adv Wound Care 1999; 12: 22-30.

17. Lipshitz D. Valoración nutricional del adulto mayor. En: OPS, OMS. editores Modelo de la valoración clínica. OPS/OMS; 1994. p. 58-70.

18. Albrecht R. Cuidados hospitalarios en el Adulto Mayor. URL disponible en: http:// www.prodigyweb.net.mix/rolfi/page5.htm (Fecha de acceso: 15 de Julio de 2006)

19. Casimiro C, García de Lorenzo A, Usan L. Prevalence of decubitus ulcer ad associated risk factors in an institutionalized spanish elderly population. Nutrition 2002; 18: 408-14.

20. Varela L, Chigne O. Ulceras por presión. En: Varela L. Principios de Geriatría y Gerontología. Lima. Editorial UPCH; 2003. p. 247-52.

21. Souza D, Gouveia V. Factores de riesgo para el desarrollo de úlceras por presión en adultos mayores atendidos en asilo. Rev Latino Am Enfermagem 2007; 15: 958-64.

Recibido: 23/03/08

Aceptado para publicación: 19/02/09 